\title{
Is Multimedia Convergence To Be Welcomed?
}

\author{
John Thanassoulis* \\ Oxford University
}

November 2008

\begin{abstract}
This paper considers the consumer implications of the process of convergence across multimedia and telecoms markets. Convergence begins when one firm begins to sell products in hitherto separate horizontal markets competing against rivals active in just one or other of the markets. Convergence creates a strategic link between the markets which alters the price levels; creates the possibility of bundle prices; and creates winners and losers in the population. Partial convergence (e.g. a merged provider of telephony and internet services vs. independent sellers of telephony or internet broadband) lowers prices in the less competitive sector, raises them in the more competitive sector and raises the total prices paid by consumers active in both sectors as compared to the counter-factual of no convergence. Full convergence (e.g. multiple firms offering TV and internet bundles) leads to deep discounts for bundle purchases but no reductions in stand alone prices paid by consumers in only one of the converging sectors.
\end{abstract}

Keywords: Multimedia; Consumer Surplus; Bundling; Pay TV; Internet; Telecommunications JEL Classification D43, L13

\section{Introduction}

Across the globe the process of multimedia convergence is very much in the public eye. Sky (part of Rupert Murdoch's News Corporation) and Virgin are two UK companies of substantial size which have recently begun competing aggressively head-to-head in multiple technology markets. Figure 1 illustrates the resultant triple-play offers including broadband, TV and fixed telephony at bundle discounts. Comcast of the US is following the same strategy. In other countries

\footnotetext{
${ }^{*}$ I would like to thank seminar participants at the UK's Office of Communications Regulation (OFCOM) and
} the University of Oxford. All errors remain my own. 
this process of convergence is yet to begin or only partially complete. This paper asks which consumers are the winners and which the losers from this convergence process. Further, is convergence inevitable?

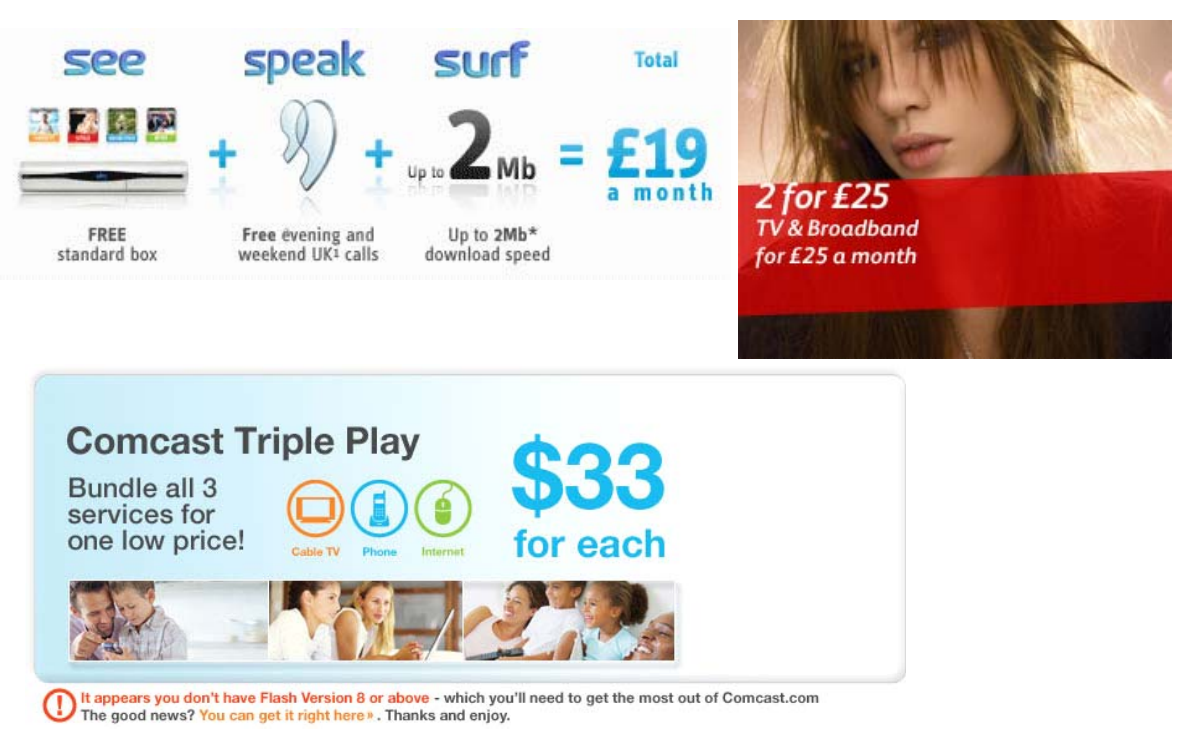

Figure 1: Adverts for bundles from the main two providers of pay TV and broadband in the UK: Sky and Virgin Media. Comcast in the US offers a similar package.

Convergence occurs when one firm, through merger or organic growth, begins to sell products in hitherto separate horizontal markets. An example would be a provider of Pay TV services acquiring a local telephony company or an internet provider. By converging the merged firm is in a position to strategically link the pricing between markets: adjusting the price levels and introducing bundle reductions.

Initially the merged firm may be competing against rivals who have yet to converge. I refer to this as a situation of partial convergence. If full convergence is reached then firms with full product lines compete head-to head with their mixed bundle offers (Figure 1).

In building a model to understand the implications of convergence I explicitly note that the main source of differentiation is often at the firm level - and not at the product level. For example, in the case of convergence of TV and internet any given channel, such as CNN, appears the same on any firm which carries it. The same is broadly true for broadband internet access. However one firm may have better customer service or take less time to organise engineers. This is firm level differentiation. As differentiation is between firms, a consumer buying more than one product from the same firm enjoys economies of scope. For example, buying pay $\mathrm{TV}$ and internet broadband from one provider allows one to deal with just one customer service 
department, receive one bill, establish one standing order, and have one process for the resolution of complaints.

To allow distributional effects of convergence to be most readily understood the model focuses on two types of consumer. Firstly there are 'small' consumers who are only interested in a subset of the products being converged: they are either in the market for good $X$ or good $Y$. These separate markets may not be equally competitive. Secondly there are 'large' consumers who desire the whole set of products being converged: both $X$ and $Y$. Large consumers harness economies of scope if they go to only one firm. Thus a merged firm can seek to target large and small consumers differentially using mixed bundle tariffs or by adjusting relative price levels; while independent rivals have fewer tools with which to respond to diverse potential consumers.

The analysis reveals that partial convergence lowers the consumer surplus of the 'small' customer who seeks to buy only the more competitive of the components. Partial convergence (a merged firm selling $X$ and $Y$ competing with separate $X$ and $Y$ sellers) links a more competitive good $X$ with a relatively less competitive good $Y$. The strategic interaction created by the partial convergence ensures that the price of good $X$ when sold alone rises. The price of the bundle of both products also rises - so the large buyers pay more under partial convergence than they did pre-convergence. However these consumers benefit from the economies of scope as they save on hassle and time costs. Consumers of good $Y$ only (the less competitive good) are winners from partial convergence: their prices fall from all providers. Independent sellers of good $Y$ wish to keep large and small consumers. But the large consumers, the ones buying both goods, see that $X$ is more expensive and so the set of both would be too unless the independent $Y$ seller responds by lowering her price. Independent sellers of $Y$ are therefore forced to lower their prices to mitigate the $X$ price rise which pulls the price of component $Y$ down across the industry. The profits of the unmerged good $Y$ seller fall as a result of partial convergence. Should the business involve substantial fixed costs this could force the unmerged firm out of business and so constitutes a theory of potential harm.

Only once full convergence is attained do the large buyers (purchasers of both goods $X$ and $Y$ ) enjoy serious deep bundle discounts - their consumer surplus rises. However the small buyers see their prices revert to pre-convergence levels. By using sophisticated bundle tariffs the competing firms can separate the large from the small consumers. Competition is intense for the large consumers, but reverts back to its previous intensity for the small guy. Hence if you were a consumer of good $Y$ only, benefiting from lower prices from partial convergence, these gains are removed upon full convergence. But overall compared to the pre-convergence case, full convergence is to be welcomed: at least large buyers gain even if small buyers stand still. 
The model is presented in Section 2; Section 3 analyses the process of convergence and Section 4 extends the model to endogenise the process of convergence and probe the model's robustness. In Section 5 I offer a short case study of the multimedia market in the UK and outline the distributional implications of the current levels of convergence.

There is, to my knowledge, only one paper which offers some insights for the process of convergence (Nalebuff 2000) - and then only when differentiation is at the product and not at the firm level. There are only slightly more papers which consider bundling when differentiation is at the firm and not product level and these are all silent about the distributional impact of convergence focusing instead on competitive price discrimination under full convergence (Thanassoulis (2007), Armstrong and Vickers $(2001,2008)$ ). I explore how the contribution here relates to these works and augments the wider knowledge about bundling in Section 6. Section 7 concludes with all technical proofs contained in the appendix.

\section{The Model}

Consider a process of convergence in the market for two goods: $X$ and $Y$. Firms $I X$ and $I I X$ both sell good $X$ only which they can produce at the same constant marginal cost. Firms $I Y$ and $I I Y$ both sell good $Y$ only, with again neither having an a priori cost advantage over the other. We will analyse three different ownership structures for these 4 firms. The first will be the pre-convergence case with all four firms competing separately. Next we will analyse partial convergence in which firms $I I X$ and $I I Y$ have merged to form firm $I I$ which competes with separate firms $I X$ and $I Y$. Finally we will analyse full convergence in which $I X$ and $I Y$ also merge to form firm $I$ which competes with the (merged) firm $I I$.

Firms are assumed to sell identical goods $X$ and $Y$ - however, the firms themselves are differentiated. In the case of multimedia this is an accurate description for many sectors which are converging. The introduction gave an explicit example in the case of TV: CNN for example is identical on rival platforms, but firms are differentiated by items such as their customer service, speed of engineer callout, clarity of bill etc. Therefore consumers incur some utility cost in visiting either seller, but this cost does not grow proportionately with extra purchases.

To study the effect of convergence on consumer surplus generally we introduce three different types of consumer. A proportion $A X$ of consumers are only in the market for one unit of good $X$. These consumers are modelled using the standard Hotelling approach. Thus consumers $A X$ are uniformly distributed along a Hotelling line of length 1 with firm $I X$ located at 0 and firm $I I X$ at 1 . The strength of competition in the good $X$ market is captured by the taste parameter $\lambda_{X}$. For a consumer located at $\theta$, an $A X$ consumer who buys from good $I I X$ incurs a taste cost 
of $\lambda_{X}(1-\theta)$.

The proportion $A Y$ of consumers who desire one unit of good $Y$ are modelled similarly with sellers $I Y$ and $I I Y$ located at opposite ends of the Hotelling line. $\lambda_{Y}$ captures the strength of competition in the good $Y$ market. We assume that $\lambda_{X} \leq \lambda_{Y}$ so that good $X$ is the more competitive of the two markets (the firms are seen as closer substitutes).

All other consumers, a proportion $B$, desire the bundle of both good $X$ and good $Y$. These are the 'large' consumers who purchase both of the converging technologies. The $B$ consumers have tastes for good $X$ and $Y$ which are distributed as the $A X$ and $A Y$ consumers. Thus a $B$ consumer is modelled as having location $\theta_{X}, \theta_{Y}$ with each parameter uniformly distributed. In the pre-convergence case therefore, a type $B$ consumer who buys component $I X$ as part of her bundle incurs taste cost of $\lambda_{X}$ times $\theta_{X}$ : the distance travelled to firm $I X$.

Without loss of generality, by a relabelling if necessary, we have positive correlation between $\theta_{X}$ and $\theta_{Y}$. That is those who like the customer service of $I X$ also like that of $I Y$, and similar for $I I X$ and $I I Y$. I make the simplifying assumption that this correlation is perfect allowing $B$ consumers' tastes to be captured by just one location variable: $\theta$. Convergence begins when $I I X$ and $I I Y$ merge to form $I I$ leaving the $B$ consumers with the choice depicted in Figure 2.

This modelling choice yields a tractable problem whose solution generates some welfare insight. The assumption that $I I X$ should merge with $I I Y$ is motivated by the observation that firms contemplating merger are likely to prefer merger partners whose products are attractive to their core customers. Alternatively this assumption can be motivated by the observation that after a merger the converged firm is likely to reposition her products to appeal to a core set of customers; while the independent rivals will optimally respond by differentiating themselves from the merged entity.

Consumers enjoy economies of effort or scope in buying from only one firm. The taste cost parameter for a consumer buying two goods from one firm is denoted $\lambda_{B}$ and we assume that $\lambda_{B}<\lambda_{X}+\lambda_{Y}$. Figure 2 depicts a $B$ type consumer choosing between going to the merged firm $I I$ versus buying $X$ from $I X$ and $Y$ from the independent $I Y$.

Finally we assume that the merger does not make the firm specific disutility any worse than either of the two merging parties. That is we have

$$
\lambda_{B}=\max \left\{\lambda_{X}, \lambda_{Y}\right\}
$$

This would be so in the case of TV/internet if, for example, convergence via merger does not increase the length of time consumers are on hold when calling customer services; or increase the length of delay before an engineer is dispatched. Section 4 relaxes this assumption. 


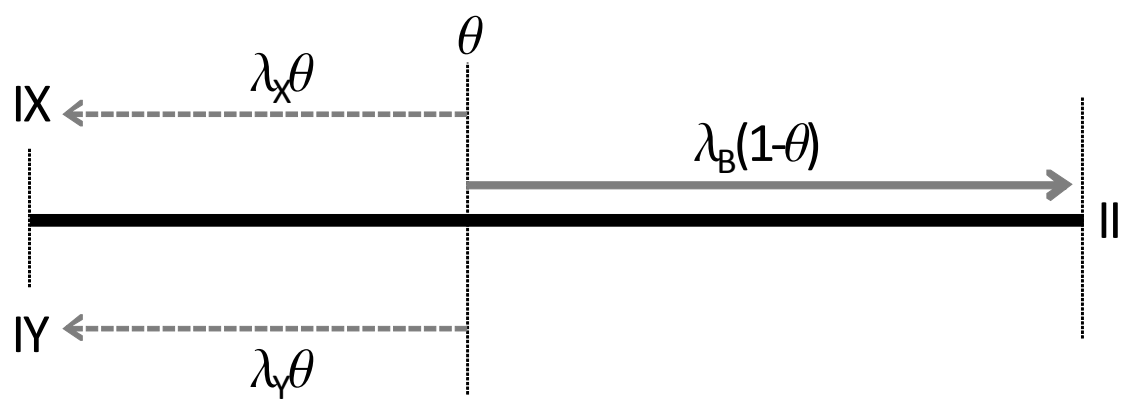

Figure 2: A depiction of the type $B$ consumers who desire both products $X$ and $Y$. If these consumers go to a merged firm then they save on taste costs: $\lambda_{B}$ times distance as opposed to $\lambda_{X}$ plus $\lambda_{Y}$ times distance if the goods are purchased from two separate firms.

\section{Analysis Of The Process Of Convergence}

\subsection{The Pre Convergence Case}

As a benchmark we analyse the market outcomes before any convergence has occurred. $X$ and $Y$ are separate markets. Large $B$ type buyers will take part in each market and there is no strategic link between the markets. We therefore have standard Hotelling competition for both $X$ and $Y$. The following result is therefore derived by standard analysis (so its proof is omitted):

Proposition 1 In the pre convergence case there is a unique equilibrium. In this equilibrium:

1. Firms $I X$ and IIX charge a margin of $\lambda_{X}$ over cost. Firms $I Y$ and IIY charge a margin of $\lambda_{Y}$ over costs. Consumers split themselves between the firms symmetrically as prices are equal.

2. Type $B$ consumers pay a margin of $\lambda_{X}+\lambda_{Y}$ above cost.

3. Firm IX makes a profit of $(A X+B) \frac{1}{2} \lambda_{X}$. Similarly for the other firms.

\subsection{Partial Convergence}

Suppose that $I I X$ and $I I Y$ have merged to form firm $I I$. This firm has converged - but is in competition with separate firms $I X$ and $I Y$. We denote the margin above cost charged for $X$ by firm $I X$ as $\rho_{X}$, while $I Y$ charges margin $\rho_{Y}$. Partial convergence provides the potential for firm $I I$ to offer a bundled tariff whereas the competition cannot. The merged firm $I I$ therefore sells $X, Y$, or the bundle of both goods at margins $\{x, y, b\}$. 
Given margins set by the firms, the indifferent $A X$ type consumer will be located at $\hat{\theta}_{X}$, and similarly the indifferent $A Y$ type consumer at $\hat{\theta}_{Y}$ where

$$
2 \lambda_{X} \hat{\theta}_{X}=\lambda_{X}+x-\rho_{X} \text { and } 2 \lambda_{Y} \hat{\theta}_{Y}=\lambda_{Y}+y-\rho_{Y}
$$

A $B$ consumer who goes to $I I$ to buy both goods gains an economy of scale by incurring transport cost proportional to $\lambda_{B}=\max \left\{\lambda_{X}, \lambda_{Y}\right\}$. To facilitate the determination of the equilibrium we begin by working under the following assumption.

No-hybrids assumption In equilibrium the 'large' $B$ type consumers either go to $I I$ or buy the set $\langle I X, I Y\rangle$.

Thus in equilibrium (and in small deviations around equilibrium) we start by assuming that the consumers do not form hybrid bundles combining a product from $I I$ and a product from either $I X$ or $I Y$. We will subsequently drop this assumption and show that it is without loss of generality - there can be no equilibrium in this model in which type $B$ consumers strictly prefer to form hybrid bundles. Under the no hybrids assumption the marginal type $B$ consumer between $I I$ and $\langle I X, I Y\rangle$ is at $\hat{\theta}_{B}$ where

$$
\hat{\theta}_{B}\left(\lambda_{X}+\lambda_{Y}+\lambda_{B}\right)=\lambda_{B}+b-\rho_{X}-\rho_{Y}
$$

The market shares can be used to derive the firms' profit functions:

$$
\begin{aligned}
\Pi_{I I}(x, y, b) & =A X \cdot x\left(1-\hat{\theta}_{X}\right)+A Y \cdot y\left(1-\hat{\theta}_{Y}\right)+B \cdot b\left(1-\hat{\theta}_{B}\right) \\
\Pi_{I X}\left(\rho_{X}\right) & =\rho_{X}\left[A X \cdot \hat{\theta}_{X}+B \cdot \hat{\theta}_{B}\right]
\end{aligned}
$$

The profit function for $I Y$ is analogous to $\Pi_{I X}$. The firms seek to maximise their profits. The strategic competition can be solved to yield:

Lemma 1 Under the no-hybrids assumption the price equilibrium of the market is given by:

1. Firm II does not offer a special bundled tariff and sets margins equal to

$$
\begin{aligned}
x & =\frac{1}{2}\left(\lambda_{X}+\rho_{X}\right) ; y=\frac{1}{2}\left(\lambda_{Y}+\rho_{Y}\right) \\
b & =\frac{1}{2}\left(\lambda_{X}+\lambda_{Y}+\rho_{X}+\rho_{Y}\right)=x+y
\end{aligned}
$$

2. Firms $I X$ and $I Y$ set margins equal to

$$
\left(\begin{array}{c}
\rho_{X} \\
\rho_{Y}
\end{array}\right)=\left(\begin{array}{c}
\lambda_{X} \\
\lambda_{Y}
\end{array}\right)+\frac{\beta\left(\lambda_{Y}-\lambda_{X}\right)}{\frac{9}{4}\left(\alpha_{X} \alpha_{Y}+\beta\left(\alpha_{X}+\alpha_{Y}\right)\right)+2 \beta^{2}} \cdot\left(\begin{array}{c}
\frac{3}{2}\left(\alpha_{Y}+\beta\right) \\
-\frac{1}{2} \beta
\end{array}\right)
$$

where

$$
\alpha_{X}=\frac{A X}{2 \lambda_{X}} ; \alpha_{Y}=\frac{A Y}{2 \lambda_{Y}} ; \beta=\frac{B}{\lambda_{X}+2 \lambda_{Y}}
$$


Proof. See Appendix for all proofs.

It may appear surprising that the merged entity $(I I)$ does not seek to offer a price discount for bundle good consumers. The result that bundle discounts are either non existent or small in the case of partial convergence is general - bundle discounts will be a much more important feature of full convergence. The fact that the discount is exactly zero here is an implication of the Hotelling model with uniform consumer dispersion.

To see the intuition behind the result note that as type $B$ consumers do not form hybrids (initially by assumption) the price charged by $I I$ for the bundle (b) has no cannibalising effect on the sales of goods $X$ and $Y$ individually. Thus the prices $x$ and $b$ serve two different populations and these prices can be set with reference to the elasticities of demand of each of these populations. Take as a benchmark that $I I$ offers no bundle reduction and sets $b=x+y$. By virtue of the economies of scope $B$ consumers enjoy in buying from a merged firm, $I I$ will serve a greater proportion of large consumers than it does of the small $A$ type consumers. This will act to lower the elasticity of demand of the $B$ consumers encouraging a higher bundle price as any price reductions are felt on a larger volume. However a small price reduction wins sales of both components $X$ and $Y$ from large consumers, and not just one component. Thus the extra profit made on new sales is much larger for $B$ than for small $A$ type consumers. These two effects work in opposite directions and so keep any bundle price reduction small. In the case of the standard Hotelling model with uniform consumer demand these two effects exactly offset each other and so the bundle price exactly equals the sum of the pure component prices.

Note that though prices are additive, the large $B$ type consumers perceive a sub-additive bundle overall as they incur lower taste/transport costs from the bundle purchase. Lemma 1 makes the point that if a large converged firm is competing with independent rivals when differentiation is at the firm level, then the economies of scope generated for the consumer by dint of convergence are sufficient to maximise profits. There is no need to reduce value by introducing large bundle price reductions. ${ }^{1}$

To complete our analysis of the partial-convergence market outcomes we now drop the nohybrid assumption. The economies of scope $B$ consumers enjoy in buying both their component products from one firm ensure that the analysis is unchanged:

\section{Lemma 2 Under partial convergence:}

\footnotetext{
${ }^{1}$ We have made the simplification that small $A X, A Y$ consumers cannot be upgraded to large $B$ type consumers. In a richer model with a continuum of valuations for the products, a seller could offer volume discounts to induce $A X$ types at the margin to become large $B$ type consumers. The volume discount motivation is well understood (Mussa and Rosen 1978 and the monopoly price discrimination literature resulting) and operates independently of the strategic environment.
} 
1. There can be no equilibrium in which $B$ consumers strictly prefer to form hybrid bundles combining goods from II with IX or IY.

2. Any interior equilibrium must satisfy Lemma 1.

3. An interior equilibrium exists at least locally if the margins captured in (7) satisfy

$$
\left(2 \lambda_{Y}-\lambda_{X}\right) \rho_{X}-2 \lambda_{X} \rho_{Y}<\lambda_{X}^{2}
$$

This condition is satisfied in the special case of the component good markets being equally competitive $\left(\lambda_{X}=\lambda_{Y}\right)$.

The fact that large $B$ consumers choose not to form hybrid bundles is not surprising. Multimedia markets are often casually referred to as one-stop shopping markets. This model generates one-stop shopping behaviour endogenously. It is possible for the market outcome to be a corner solution however in which prices adjust so that $B$ consumers are indifferent between forming a hybrid bundle and buying both goods at $I I$. Condition (9) gives a sufficient condition for this not to be the case. In the rest of the paper I restrict attention to parameters which allow for a non-corner (interior) equilibrium - this includes the special case of the $X$ and $Y$ markets being equally competitive $\left(\lambda_{X}=\lambda_{Y}\right)$.

\subsubsection{Market Analysis}

The distributional and profit effects of moving from no convergence to partial convergence can now be established:

Proposition 2 Comparing partial convergence achieved by the merger of II to the no convergence benchmark we have:

\begin{tabular}{|c|c|c|c|}
\hline & $A X$ consumers & $A Y$ consumers & $B$ consumers \\
\hline Prices & $\uparrow$ & $\downarrow$ & $\uparrow$ \\
\hline Firm II market share & $\uparrow$ & $\downarrow$ & $\uparrow$ \\
\hline & [more competitive sector] & [less competitive sector] & \\
\hline
\end{tabular}

And partial convergence lowers the profits of (unmerged) firm IY.

Proposition 2 shows that the strategic link created by partial convergence between markets $X$ and $Y$ creates winners and losers in the population. Partial convergence leads to lower consumers surplus (higher prices) for consumers in the more competitive sector, coupled with higher consumer surplus (lower prices) for consumers in the less competitive sector. The large $B$ type consumers see higher retail prices whomever they buy from. These higher prices will be 
in part offset by the reductions in the taste cost which the merger has afforded for those who buy from $I I$. Overall the consumer surplus outcome for $B$ consumers is ambiguous. ${ }^{2}$ The rest of this section tries to explain the economic intuition behind these outcomes.

We begin our analysis of the strategic effects of partial convergence by first turning to firm $I X$ which sells the more competitive of the two goods. As a result of the merger creating firm $I I, B$ consumers who purchase from $I X$ have a lower elasticity of demand than the $A X$ consumers $\left(\lambda_{B}=\lambda_{Y} \geq \lambda_{X}\right)$. If they were to transfer to buying from $I I$ then they would, given the economies of scope, also move their good $Y$ purchases to $I I$. Thus the $B$ type consumers see a greater differentiation between $\langle I X, I Y\rangle$ and $I I$ than the small $A X$ consumers see between $I X$ and $I I$. Therefore the partial convergence has lowered the aggregate elasticity of demand faced by firm $I X$ and so she would raise her price. This price rise leads firm $I I$ to also raise its component $X$ price, though not by as much. Thus $A X$ consumers pay more and $I I$ wins a greater market share here even though it raises its price to these consumers.

Next consider firm $I Y$. Both $B$ and $A Y$ consumers incur the same taste cost in moving to $I I$ due to the fact that $Y$ was the less competitive product and type $B$ consumers harness economies of scope in going to $I I\left(\lambda_{B}=\lambda_{Y}\right)$. However the competitive position of $I Y$ 's product as regards the large $B$ consumers has been damaged by $I X$ 's price rise. As $B$ consumers who buy from $I Y$ also buy from $I X$, and $I X$ has raised her price, the demand from $B$ consumers is diminished. To counter this effect $I Y$ must counteract some of the price rise from $I X$ by lowering her price. She doesn't counteract it all as such a price drop is loss making for the small $A Y$ type consumers. On balance therefore $I Y$ lowers her price, but not by as large a margin as $I X$ raised hers.

Returning to firm $I I$, the $A Y$ consumers see a price reduction from firm $I Y$ and so $I I$ responds by lowering her component $Y$ price. Thus $A Y$ consumers benefit. The sum of the prices from $I X$ and $I Y$ has risen above the pre-convergence level. Therefore $I I^{\prime} s$ optimal response is to also allow her bundle price to rise. Therefore type $B$ consumers pay higher prices, however those that buy from $I I$ save taste costs (harness convenience savings) from the economies of scope arising from the fact that we have firm specific (rather than product specific) differentiation.

Finally the strategic link between markets $X$ and $Y$ has forced firm $I Y$ to lose market share amongst the large $B$ buyers, and to lower the prices charged to $A Y$ buyers so that profits unambiguously decline. If $I Y$ incurs large fixed costs of operation then clearly $I Y$ could be driven out of business by the merger of $I I$.

\footnotetext{
${ }^{2}$ There are parameter values for which the consumer surplus of $B$ consumers falls, and different ones for which they rise. See Figure 3.
} 


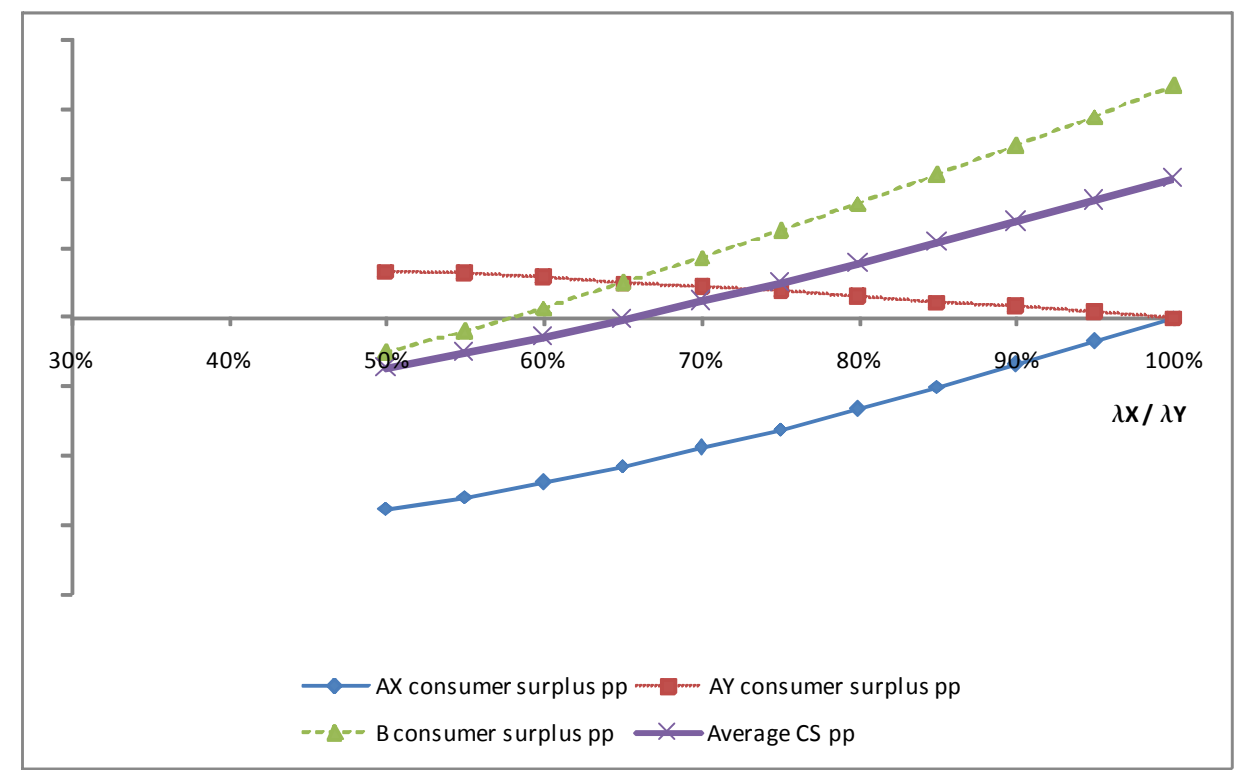

Figure 3: A graph of the consumer surplus changes from partial convergence as compared to the benchmark of no convergence. The proportions of consumers assumed for this numerical simulation were $A X=0.2=A Y, B=0.6$. For a range of values of $\frac{\lambda_{X}}{\lambda_{Y}}$ lying within $\left(0, \frac{1}{2}\right)$ condition (9) is broken implying that the equilibrium is given by a corner solution.

Figure 3 explores one numerical example and analyses the consumer surplus implications for different relative competitiveness values of markets $X$ and $Y$. The graph illustrates that large $B$ consumers can lose or gain from partial convergence in consumer surplus terms. Similarly the figure illustrates that the overall population consumer surplus effects can also either go up or down. So though partial convergence leads to winners and losers, an overall ranking between the two states is not available.

\subsection{Full Convergence}

We now consider the case of full convergence: $I X$ and $I Y$ merge to form firm $I$ selling both goods $X$ and $Y$ which competes with the merged firm $I I$. Both firms are now in a position to strategically link the two markets. As a result we will see that both firms will offer deeply discounted bundle prices to those buying both goods:

Lemma 3 The unique symmetric equilibrium in the case of full convergence is given by both I and II setting margins

$$
x=\lambda_{X}, y=\lambda_{Y}, b=\lambda_{B}\left(:=\lambda_{Y}\right)
$$


Under full convergence the competing firms are in a position to sub-additively bundle and so target different consumer groups with different prices. The $B$ consumers do not form hybrids due to the economies of scope that they enjoy by purchasing both products from a single firm; so the three consumer types are strategically separated. The small $A X$ and $A Y$ consumers therefore receive the pre-convergence price. The greater the economies of scope $B$ consumers harness in purchasing from one firm, the less differentiated the competing firms seem to these consumers to be. This is because the difference in the disutility of using a firm for one product versus both is smaller if the economy of scope is large. This acts to push the bundle price down below the sum of the component prices. Under condition (1) generated by assuming that merger does not damage a firm beyond the worse of the merger partners, the more competitive of the goods $(X)$ is sold as an upgrade at cost (with no added margin) if the less competitive good $(Y)$ is purchased. Lemma 3 allows the full, partial and pre convergence scenarios to be compared:

Proposition 3 Comparing all three possible convergence scenarios:

\begin{tabular}{|c|c|c|c|}
\hline & AX consumers & AY consumers & $B$ consumers \\
\hline \multirow{5}{*}{ Prices } & partial & pre $=$ full & partial \\
& $\vee$ & $\vee$ & $\vee$ \\
& pre $=$ full & partial & $\vee$ \\
& & full \\
\hline \multicolumn{2}{|r|}{} \\
Where pre=pre convergence, partial=partial convergence and full=full convergence.
\end{tabular}

This result follows from Lemmas 1 and 3 as well as Proposition 1. Full convergence benefits large $B$ consumers as compared to the pre-convergence benchmark. However small consumers gain nothing once convergence has run its course as the mixed bundled tariffs separates these consumers from the large $B$ type. The interim of partial convergence leads to bundle prices rising and the price of hitherto competitive components rising also as compared to the preconvergence benchmark. However recall that bundle consumers gain through the convenience of having one vendor (lower incurred transport costs) so that their overall surplus change is ambiguous. The purchasers of the hitherto less competitive good are likely to see lower prices under partial convergence. 


\section{Analysis Of Model Extensions}

\subsection{Endogenising Convergence}

Is the partial convergence stage only transitory? This question is important as the main negative effects on consumers arose under partial convergence (higher prices for one component and the bundle, reduced profits for one of the unmerged rivals resulting in the possibility of predation). This section asks whether under partial convergence the unmerged rivals $I X$ and $I Y$ would decide to merge also, moving the industry to full convergence. I explore this question by endogenising the convergence process. We will see that partial convergence - with all its negative consumer surplus outcomes - is unfortunately an equilibrium outcome. The convergence process will not necessarily reach its ultimate conclusion.

Consider the following two stage game.

Stage 1. Firms $I X$ and $I Y$ decide if they wish to merge. Simultaneously firms $I I X$ and $I I Y$ also decide if they would like to merge.

Stage 2. The independent firms compete non-collusively in prices.

I restrict attention to the leading special case of goods $X$ and $Y$ being equally competitive $\left(\lambda_{X}=\lambda_{Y}:=\lambda\right)$. Lemmas 1 and 3 and Proposition 1 allow us to derive the following pay-off matrix:

II

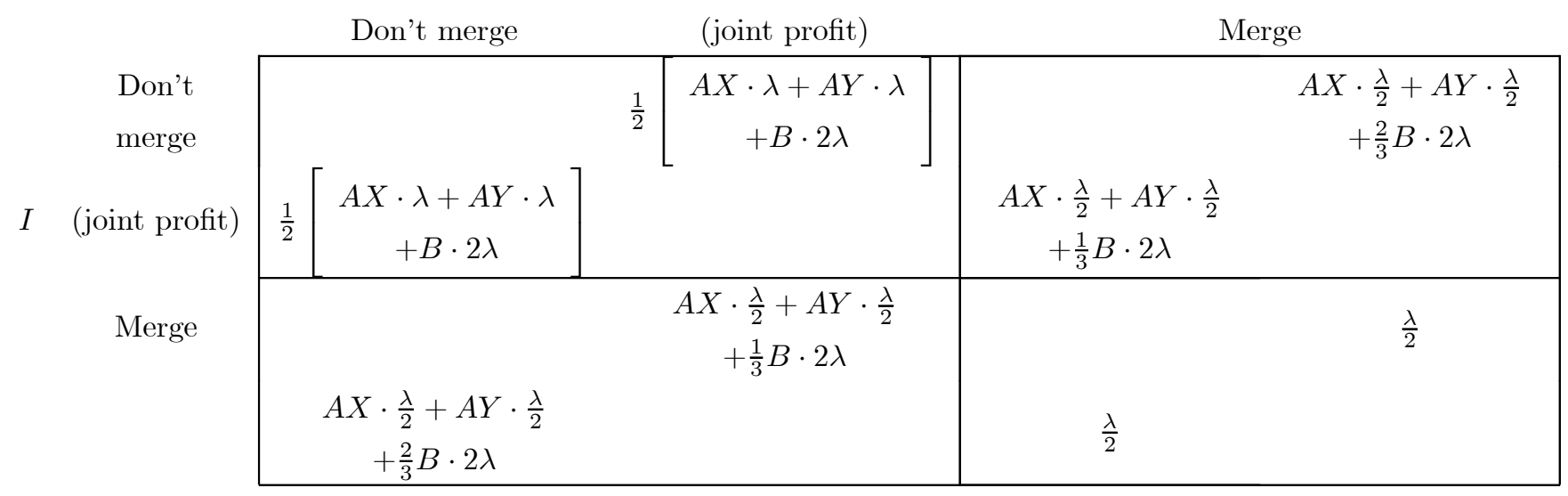

Inspection of the game matrix allows us to rank the profits of each game outcome:

$$
\begin{aligned}
{\left[\Pi_{I I}\right]_{\text {full convergence }} } & <\left[\Pi_{I I X}+\Pi_{I I Y}\right]_{\text {partial convergence }(I \text { merged })} \\
& <\left[\Pi_{I I X}+\Pi_{I I Y}\right]_{\text {no convergence }}<\left[\Pi_{I I X}+\Pi_{I I Y}\right]_{\text {partial convergence }(I I \text { merged })}
\end{aligned}
$$

Hence we have: 
Proposition 4 The two stage convergence game has one type of pure strategy equilibrium: partial convergence.

If one pair of firms merges they capture two strategic advantages over their unmerged rivals. Firstly they are able to offer large $B$ consumers economies of scope and so grow their market share amongst this group with no (or limited) price reductions. The second effect is that the merged firm can coordinate targeted pricing at the different subgroups in the population. Both of these advantages lead to the merged firm growing their profit as compared to their unmerged rivals. Hence no convergence is not a Nash equilibrium as partial convergence dominates it.

The unmerged firm would not rather merge herself in response as full convergence leads to intense bundle on bundle competition for the large $B$ buyers. The price to these large buyers therefore plummets which lowers everyone's profits. On the other hand remaining separate creates a strategic effect: as the independent firms do not internalise the other's gain from large $B$ type buyers, they do not overall price as aggressively as they would do if they merged. As competition in prices are strategic complements this also eases some of the competitive pressure created by the merged firm and so prevents profits falling to fully merged levels.

Hence partial convergence is the unique pure strategy equilibrium of the dynamic convergence game. ${ }^{3}$ As a result there are advantages in being the first firm to merge and offer a bundled proposition returning economies of scope to the large $B$ buyers.

\subsection{Multiple Products}

Suppose, more generally, that within each of markets $X$ and $Y$ there are multiple components which might be bought. This section will address how our results extend into this more complicated setting. Further, a natural question of interest is whether convergence will distort the set of products that consumers buy. This section indicates that the pricing equilibrium in this richer setting is for firms to use two part-tariffs. The variable price is set at cost while the fixed fee allows the firms to recoup margins equal to those determined in the core model. Thus we will see that convergence does not distort consumption patterns of those who purchase in equilibrium; and the consumer surplus implications of convergence remain as in the core analysis.

To develop the reported insights I augment the core model as follows. Suppose that there are $N$ different goods within market $X$. Thus a consumer's purchases can be denoted by the vector $\mathbf{X}=\left(X_{1}, \ldots, X_{N}\right)$ with $X_{i}$ denoting the quantity of good $i$ from market $X$ purchased. In the case of pay TV $X_{i}$ would be one of the channels which could be purchased. We suppose

\footnotetext{
${ }^{3}$ There is of course also a mixed strategy equilibrium. In this case there is a positive probability of miscoordination in which case full convergence can result.
} 
that consumers one-stop shop for goods within a given market, but can source from one firm for market $X$ goods and a different firm for market $Y$ goods. The cost of serving a consumer with demand $\mathbf{X}$ is

$$
k_{X}+\mathbf{c}_{X} \cdot \mathbf{X}
$$

which captures a per consumer fixed cost of $k_{X}$ plus the constant marginal cost per good selected. The costs are the same across firms. The costs differ between markets $X$ and $Y$. We allow firms to set two-part tariffs.

As before, type $A X$ consumers are uniformly distributed between $I X$ and $I I X$, and incur taste costs proportional to $\lambda_{X}$ in purchasing from either firm. Independently of location, $\theta$, the $A X$ consumers have a second type variable: $\varphi_{X}$. This captures a preference between the types of good in market $X$. The consumer has gross utility of $U\left(\boldsymbol{\varphi}_{X}, \mathbf{X}\right)$ if she buys $\mathbf{X}$. Let $v_{A X}\left(\boldsymbol{\varphi}_{X}\right)$ denote the maximal surplus available to this consumer arising from marginal cost pricing:

$$
v_{A X}\left(\boldsymbol{\varphi}_{X}\right)=\max _{\mathbf{X}}\left\{U\left(\left(\boldsymbol{\varphi}_{X}, \mathbf{X}\right)\right)-\mathbf{c}_{X} \cdot \mathbf{X}\right\}
$$

The $A Y$ consumers are modelled similarly in their purchases from market $Y$. Type $B$ consumers buy goods from both markets $X$ and $Y$ and so have gross utility of $U\left(\boldsymbol{\varphi}_{B}, \mathbf{X}, \mathbf{Y}\right)$. The utilities of all the consumers are assumed high enough that all consumers take part by purchasing some volume of at least one good in equilibrium. This model is a natural extension of Armstrong and Vickers (2001).

Lemma 4 The margins found in the core model (Proposition 1 and Lemmas 1 and 3) are equilibria of the model with multiple goods in each market. Thus the price charged by firm IX is given by

$$
\begin{aligned}
p_{X}(\mathbf{X}) & =[\text { Fixed cost per consumer }]+[\text { Variable costs }]+[\text { margin from our core model }] \\
& =k_{X}+\mathbf{c}_{X} \cdot \mathbf{X}+[\text { margin from our core model }]
\end{aligned}
$$

And analogously for the other firms in all convergence states.

Proof. That Proposition 1 (pre-convergence) extends to this setting is a corollary of Armstrong and Vickers (2001 Proposition 5). If one assumes that $B$ consumers one-stop shop then Lemma 3 (full convergence) is also confirmed by Armstrong and Vickers (2001 Proposition 5). The equilibrium is robust to relaxing the one-stop shop assumption as the market is split equally between the two merged firms and so no $B$ consumer would prefer to form a hybrid bundle by splitting purchases between the two sellers.

For the case of partial convergence we proceed as follows. Suppose firms could observe each consumers' type, though not her location, and suppose that the firms are charging tariffs as 
in the lemma. We aim to show no firm has an incentive to deviate from the proposed tariffs. The firms are competing in utilities to attract consumers. Therefore the optimal response to the rivals' prices will be to set marginal prices at cost, thus maximising gross utility, and then extract the desired amount of surplus as a fixed fee given a desired utility level. With such a tariff the $A X$ consumers have maximal utility given by $v_{A X}\left(\boldsymbol{\varphi}_{X}\right)$ no matter who they buy from, and analogously for $A Y$ and $B$. Thus the problem is identical to the core model and so the margins offered from the core model maximise profits. Now note that these prices are independent of the observed types $\left\{\varphi_{X}, \varphi_{Y}, \varphi_{B}\right\}$ and so the result holds for all types as required.

Therefore two part tariffs prevent participating consumers having to accept sub-optimal volumes: the degree of convergence has no effect on the marginal prices. The fixed fees consumers pay however behave exactly as in the core model. Partial convergence raises the fixed fee for those buying only products $X$ (more competitive market), and lowers it for $Y$ consumers (less competitive market) and makes no difference for $B$ consumers.

\subsection{General Consumer Economies of Scope}

The analysis so far has assumed that when a firm merges it offers no worse level of service than the worse of the two merging firms: this generated condition (1). We now make the economies of scope general replacing (1) by the assumption

$$
\max \left(\lambda_{X}, \lambda_{Y}\right) \leq \lambda_{B} \supsetneqq \lambda_{X}+\lambda_{Y}
$$

The pre-convergence case is unaffected as with no converged firm no consumer is in a position to harness economies of scope. Let us therefore turn to the case of partial convergence. Here the only possible interior equilibrium remains one in which $B$ consumers do not form hybrid bundles. The more competitive good $(X)$ rises in price as does the bundle as above. However whereas before the component good $Y$ fell in price, now it doesn't fall as far and, if economies of scope are sufficiently small, the component price of $Y$ may actually rise as compared to the pre-convergence benchmark. Thus quite possibly all consumers pay more for the goods if the industry takes the form of partial convergence. We collect these results in the following proposition which is proved in the appendix.

Proposition 5 Suppose that in the case of partial convergence, the large B type consumers have economies of scope given by (11). In this case:

1. With pre-convergence Proposition 1 is unaffected.

2. Under partial convergence: 
(a) There can be no equilibrium in which $B$ consumers form hybrid bundles.

(b) Proposition 2 holds for consumers $A X$ and $B$.

(c) Prices to consumers $A Y$ are higher than given in Lemma 1 and may lie above the pre-convergence case.

3. Under full convergence, Proposition 3 holds except for the ranking of the partial convergence $Y$ prices.

Recall that for the $I Y$ firm, partial convergence has linked the two markets and so her type $B$ consumers now consider both the price of good $I Y$ and of good $I X$ when deciding whether to buy or to instead swap to the merged firm and harness the economies of scope. Firm $I X$ has raised its price understood with the same intuition as given after Proposition 2. This creates pressure on $I Y$ to lower her price so as not to lose too many $B$ consumers. This was the force described above which resulted in good $Y$ prices falling under assumption (1) on the economies of scope. However, as the economies of scope become more modest the attraction of going to the merged firm $I I$ falls. Thus firm $I Y$ needs to lower her price less to counteract the rise $I X$ has posted. If $\lambda_{B}$ becomes high enough (economies of scope small enough) then $I Y$ will find that the $B$ consumers actually have a lower elasticity of demand in moving to $I I$ than they did before partial convergence, not withstanding the rise in $I X$ prices. At this point $I Y$ is in a position to even raise price. For this reason buyers of good $Y$ only need not see their consumer surplus rise from partial convergence if the economies of scope enjoyed by the large buyers are small.

\section{Multimedia Convergence Case Study}

The UK has seen substantial convergence of technologies in the multimedia sector spearheaded by the competition between Sky (part of Rupert Murdoch's News Corporation) and Virgin. Virgin offers Premium Sport TV, broadband internet and mobile telephony whilst Sky offers Premium Sport TV and broadband. I have already argued the channels are the same on either platform: CNN looks the same via cable or satellite. ${ }^{4}$ Broadband competition is also strong due to the close homogeneity of the product. In both broadband and pay TV an engineer may be required to set up; and technical support assistance may be required during use. Therefore I would argue that the model of firm specific differentiation is applicable here with differentiation

\footnotetext{
${ }^{4}$ Both firms in the UK also offer staggered start times of movies across channels and other features. Thus, though not identical, the actual products offered remain close.
} 
being on services (such as customer support) used when buying either one or both products, rather than on the specific nature of the channel or broadband itself.

Inspection of the market for Premium Sport TV and broadband in the UK suggests that we have seen full convergence here with both Sky and Virgin going head to head. Our analysis therefore offers the following explanations and predictions:

1. Lemma 3 delivers the result that full convergence competition should give rise to substantial bundle reductions for the 'large' consumers who desire both sets of products. This is bourne out in the advertising: Figure 1. Indeed broadband internet is offered for free with some TV packages ${ }^{5}$ - a result consistent with the simple model here as the marginal cost of an extra internet user is negligible.

2. Proposition 3 predicts that for those who want just one or the other product (TV or internet) convergence does not yield savings relative to the prices achievable without convergence.

Amongst other multimedia products and in other countries the process of convergence is much less advanced. In such settings partial convergence is liable to be more realistic and so the pattern of winners and losers amongst consumers would be different; and documented by Proposition 3.

\section{Convergence and the Bundling Literature}

This paper's contribution is to consider the distributional implications of the process of convergence in competitive markets; and do this while allowing for the firm specific differentiation prevalent in many multimedia markets. Much of the bundling literature has focussed on the question of how a firm which is dominant in one market can use bundling or tying activities to alter the industrial structure of a related market. Important papers in this vein include Whinston (1990), Carlton and Waldman (2002) and Nalebuff (2004). The question of bundling in competitive markets has been taken up in fewer papers, and an analysis applicable to the process of convergence is, to my knowledge, offered in only two other papers: Nalebuff (2000) and Granier and Podesta (2008).

Nalebuff (2000) considers demand for a system of products: all buyers require all components. As a result the distributional question of surplus amongst differing groups of consumer which we analyse here cannot be addressed. The Nalebuff analysis considers differentiation at the

\footnotetext{
${ }^{5}$ See http://mysky.sky.com/portal/site/skycom/skyproducts/skytv/pricesandpackages
} 
product and not firm level. This implies that consumers derive no economies by purchasing from one firm, beyond any bundled price reduction which might be offered. ${ }^{6}$ Nalebuff analyses how strategic competition would play out between a (converged) firm offering only the bundle of components against independent providers of differentiated versions of each component. The bundle seller has the profit advantage as a result of the coordination it has as to the price of the overall bundle. The rival component sellers do not internalise the losses of the other parts of the bundle they are sold with and so push prices above the merged firm, so losing market share. In our paper this result applies for the less competitive market, but not for the more competitive market where prices fall; and fall most for the independent seller. Secondly we have captured the effect on the equilibrium prices of the consumer economies of scope which the subset of buyers who buy both components receive. Thus we are able to document who the winners and losers will be from the process of convergence.

Granier and Podesta (2008) continue the investigation in Nalebuff (2000) by also focusing on product (not firm) differentiation and assuming all consumers require the bundle. They extend the analysis to allow consumers to differ in their correlation between valuations for the two goods.

The economic literature has more to say if full convergence is reached in an industry. Bundling between duopolists in the case of product specific, rather than firm specific, preferences are analysed in among others, Matutes and Regibeau (1992) and Reisinger (2006). However these papers assume that all consumers buy the bundle preventing a distributional analysis between different consumer groups. Further differentiation is at the product level so that the economies of scope inherent in the convergence processes we have discussed cannot be captured.

An interesting question which the literature has considered is whether, once full convergence is reached and bundle discounts become prevalent, consumer surplus would be improved by requiring firms to price à la carte? This is a particularly pertinent question in the US where high profile politicians have called for TV providers to unbundle their channels. ${ }^{7}$ Thanassoulis (2007) considers this question for markets such as pay TV ones in which consumers one stop shop as differentiation is at the firm level. Thanassoulis (2007) shows that, in the full convergence setting, à la carte pricing would increase consumer surplus as essentially large buyers provide price protection for the small buyers. Once mixed bundling is allowed this price protection is lost

\footnotetext{
${ }^{6}$ The model is motivated by Microsoft Office rather than the multimedia examples we discuss.

${ }^{7}$ When discussing the lack of à la carte pricing in cable TV, Senator John McCain noted: "When I go to the grocery store to buy a quart of milk, I don't have to buy a package of celery and a bunch of broccoli. I don't like broccoli." Washington Post, March 26, 2004, "Sorry - No a la Carte Cable."
} 
and the small buyer sees a price hike, while the large buyer enjoys low bundle prices. This price hike is large enough to lower consumer surplus overall. ${ }^{8}$ An empirical analysis of the comparison of bundling to à la carte pricing in the context of US TV is being undertaken by Crawford and Yurukoglu (2007). These authors confirm that consumer welfare would increase from pricing channels individually (as long as channels are not subsequently removed).

\section{Conclusions}

Is multimedia convergence to be welcomed? From a starting position of no convergence at all then full convergence is to be welcomed. Those consumers who desire both of the goods being converged (TV and internet, or TV and mobile telephony for example) enjoy deep price reductions on the bundle. These consumers also benefit from the economies of scope inherent in sourcing from just one firm. The 'small' consumers who desire only a subset of the converged products do no better, and no worse, than the no convergence case. Thus these consumers are left behind, but as the 'large' consumers gain, a gain is recorded overall. If à la carte pricing were mandated in the context of full convergence then even small consumers would benefit and all prices would come down further (Thanassoulis 2007).

Unfortunately the process of convergence is liable to stop at the point of partial convergence: one merged firm competing with non-merged rivals. This is exactly because full convergence raises consumer surplus by lowering profits. If a market stops at partial convergence then the whole convergence process is at best a mixed bag and can certainly be overall bad for consumers as compared to no convergence at all. Under partial convergence the prices paid by 'large' consumers who want all the converging products rise. This is so whether these buyers go to the merged firm or the independent competitors. The market share of the merged firm rises amongst this group of buyers as they harness the economies of scope available from purchasing from just one firm. Overall therefore the consumer surplus effect on these consumers is ambiguous, even though they pay more.

Partial convergence creates a means to link a more competitive market with a less competitive one. As a result prices for the more competitive good rise. Consumers who only wish to purchase this good see their consumer surplus decline. Prices for the less competitive good are pulled down. So 'small' consumers of one of the converging goods lose out while small consumers for the other good gain. Therefore whether convergence is to be welcomed will depend upon whether

\footnotetext{
${ }^{8}$ Armstrong and Vickers (2008) extend the analysis of Thanassoulis (2007) to the case of general consumer demand as opposed to large versus small buyers. The results are all confirmed in this richer setting; though the identity of the winners and losers is now made less transparent.
} 
there is a reason to favour one type of consumer to another.

\section{A Proofs From Core Model}

Proof of Lemma 1. Firm $I I^{\prime} s$ profit function is given by (4). Note that the terms in $x, y$ and $b$ are independent of eachother as no $B$ types ever buy hybrid bundles including $I I$ 's products by assumption. Hence the profit function is a negative quadratic in its margin terms $\{x, y, b\}$ which can therefore be maximised to give

$$
x=\frac{1}{2}\left(\lambda_{X}+\rho_{X}\right) ; y=\frac{1}{2}\left(\lambda_{Y}+\rho_{Y}\right) ; b=\frac{1}{2}\left(\lambda_{X}+\lambda_{Y}+\rho_{X}+\rho_{Y}\right)=x+y
$$

Therefore there is no sub-additive bundling here as no consumers make hybrid purchases.

Now consider $I X$ whose profit function is given by (5). This is a negative quadratic in $\rho_{X}$ and so the first order condition for the maximal point is

$$
\begin{aligned}
0 & =\frac{A X}{2 \lambda_{X}} \cdot\left(\lambda_{X}+x-2 \rho_{X}\right)+\frac{B}{\lambda_{X}+\lambda_{Y}+\lambda_{B}} \cdot\left(\lambda_{B}+b-2 \rho_{X}-\rho_{Y}\right) \\
& =\frac{A X}{2 \lambda_{X}} \cdot \frac{3}{2}\left(\lambda_{X}-\rho_{X}\right)+\frac{B}{\lambda_{X}+\lambda_{Y}+\lambda_{B}} \cdot\left(\lambda_{B}+\frac{1}{2}\left(\lambda_{X}+\lambda_{Y}\right)-\frac{3}{2} \rho_{X}-\frac{1}{2} \rho_{Y}\right)
\end{aligned}
$$

where we have substituted in for $x$ and $b$. Using the change of notation

$$
\alpha_{X}:=\frac{A X}{2 \lambda_{X}} \text { and } \beta:=\frac{B}{\lambda_{X}+\lambda_{Y}+\lambda_{B}}
$$

we have the system of equations (for firms $I X$ and $I Y$ )

$$
\left[\begin{array}{cc}
\frac{3}{2}\left(\alpha_{X}+\beta\right) & \frac{1}{2} \beta \\
\frac{1}{2} \beta & \frac{3}{2}\left(\alpha_{Y}+\beta\right)
\end{array}\right]\left(\begin{array}{c}
\rho_{X} \\
\rho_{Y}
\end{array}\right)=\left(\begin{array}{c}
\frac{3}{2} \lambda_{X} \alpha_{X}+\beta\left(\lambda_{B}+\frac{1}{2} \lambda_{X}+\frac{1}{2} \lambda_{Y}\right) \\
\frac{3}{2} \lambda_{Y} \alpha_{Y}+\beta\left(\lambda_{B}+\frac{1}{2} \lambda_{X}+\frac{1}{2} \lambda_{Y}\right)
\end{array}\right)
$$

These can be solved to give

$$
\left(\begin{array}{c}
\rho_{X} \\
\rho_{Y}
\end{array}\right)=\frac{\left[\begin{array}{cc}
\frac{3}{2}\left(\alpha_{Y}+\beta\right) & -\frac{1}{2} \beta \\
-\frac{1}{2} \beta & \frac{3}{2}\left(\alpha_{X}+\beta\right)
\end{array}\right]\left(\begin{array}{c}
\frac{3}{2} \lambda_{X} \alpha_{X}+\beta\left(\lambda_{B}+\frac{1}{2} \lambda_{X}+\frac{1}{2} \lambda_{Y}\right) \\
\frac{3}{2} \lambda_{Y} \alpha_{Y}+\beta\left(\lambda_{B}+\frac{1}{2} \lambda_{X}+\frac{1}{2} \lambda_{Y}\right)
\end{array}\right)}{\frac{9}{4}\left(\alpha_{X} \alpha_{Y}+\beta\left(\alpha_{X}+\alpha_{Y}\right)\right)+2 \beta^{2}}
$$

Focusing on the margin charged for good $X$ (the other is found by swapping the $X$ and $Y$ terms) we have

$$
\rho_{X}=\lambda_{X}+\frac{1}{\frac{9}{4}\left(\alpha_{X} \alpha_{Y}+\beta\left(\alpha_{X}+\alpha_{Y}\right)\right)+2 \beta^{2}}\left\{\begin{array}{c}
-\frac{9}{4} \lambda_{X} \alpha_{Y} \beta-2 \lambda_{X} \beta^{2}+\frac{3}{2}\left(\alpha_{Y}+\beta\right) \beta\left(\lambda_{B}+\frac{1}{2} \lambda_{X}+\frac{1}{2} \lambda_{Y}\right) \\
-\frac{3}{4} \lambda_{Y} \alpha_{Y} \beta-\frac{1}{2} \beta^{2}\left(\lambda_{B}+\frac{1}{2} \lambda_{X}+\frac{1}{2} \lambda_{Y}\right)
\end{array}\right\}
$$

Focusing on the brace term we can simplify this to

$$
\beta \alpha_{Y}\left\{\frac{3}{2} \lambda_{B}-\frac{3}{2} \lambda_{X}\right\}+\beta^{2}\left\{\lambda_{B}-\frac{3}{2} \lambda_{X}+\frac{1}{2} \lambda_{Y}\right\}
$$


We now use the fact that $X$ is the weakly more competitive market so that $\lambda_{X} \leq \lambda_{Y}=\lambda_{B}$ which gives (7).

Proof of Lemma 2. Part 1. Suppose, for a contradiction, that some type $B$ consumers strictly prefer to buy the hybrid $\langle I I X, I Y\rangle$. There are therefore two cutoffs for type $B$ consumers. Those with small $\theta$ 's buy from $I X$ and $I Y$. At $\hat{\theta}_{X}$ the $B$ consumer swaps to the hybrid $\langle I I X, I Y\rangle$. Then at $\hat{\theta}_{h}$ the consumer swaps from the hybrid to the bundle from $I I$. We have

$$
\begin{aligned}
\langle I I X, I Y\rangle \sim I I \Leftrightarrow x+\lambda_{X}\left(1-\hat{\theta}_{h}\right)+\rho_{Y}+\lambda_{Y} \hat{\theta}_{h} & =b+\left(1-\hat{\theta}_{h}\right) \lambda_{B} \\
\hat{\theta}_{h}\left(\lambda_{B}+\lambda_{Y}-\lambda_{X}\right) & =b-x-\lambda_{X}-\rho_{Y}+\lambda_{B}
\end{aligned}
$$

The equilibrium pricing behaviour under this assumption can be determined. Firm $I X$ is straightforward as this firm never sells (by assumption) part of a bundle product:

$$
\begin{aligned}
\Pi_{I X}\left(\rho_{X}\right) & =(A X+B) \rho_{X} \hat{\theta}_{X} \\
\frac{\partial \Pi_{I X}}{\partial \rho_{X}} & =\left(\frac{A X+B}{2 \lambda_{X}}\right)\left\{\lambda_{X}+x-2 \rho_{X}\right\} \Rightarrow \rho_{X}=\frac{1}{2}\left(x+\lambda_{X}\right)
\end{aligned}
$$

For firm $I I$ note that

$$
\begin{aligned}
\Pi_{I I}(x, y, b) & =A X \cdot x\left(1-\hat{\theta}_{X}\right)+A Y \cdot y\left(1-\hat{\theta}_{Y}\right)+B\left\{b\left(1-\hat{\theta}_{h}\right)+x\left(\hat{\theta}_{h}-\hat{\theta}_{X}\right)\right\} \\
& =(A X+B) \cdot x\left(1-\hat{\theta}_{X}\right)+A Y \cdot y\left(1-\hat{\theta}_{Y}\right)+B \cdot(b-x)\left(1-\hat{\theta}_{h}\right)
\end{aligned}
$$

Differentiating we have

$$
\frac{\partial \Pi_{I I}}{\partial y}=\frac{A Y}{2 \lambda_{Y}}\left\{\lambda_{Y}-2 y+\rho_{Y}\right\} \Rightarrow y=\frac{1}{2}\left(\rho_{Y}+\lambda_{Y}\right)
$$

For the bundle price we have

$$
\frac{\partial \Pi_{I I}}{\partial b}=\frac{B}{\lambda_{B}+\lambda_{Y}-\lambda_{X}}\left\{\lambda_{Y}-2 b+2 x+\rho_{Y}\right\} \Rightarrow b=x+\frac{1}{2}\left(\rho_{Y}+\lambda_{Y}\right)
$$

Note that again $b=x+y$ and so there is no bundling here. For the $x$ component we have

$$
\begin{aligned}
\frac{\partial \Pi_{I I}}{\partial x} & =\left(\frac{A X+B}{2 \lambda_{X}}\right)\left\{\lambda_{X}-2 x+\rho_{X}\right\}+\left(\frac{B}{\lambda_{B}+\lambda_{Y}-\lambda_{X}}\right)\left\{-\left(\lambda_{Y}-b+x+\rho_{Y}\right)+(b-x)\right\} \\
& =\left(\frac{A X+B}{2 \lambda_{X}}\right)\left\{\lambda_{X}-2 x+\rho_{X}\right\} \text { using }(15) \Rightarrow x=\frac{1}{2}\left(\lambda_{X}+\rho_{X}\right)
\end{aligned}
$$

Combining with (13) we therefore have

$$
x=\rho_{X}=\lambda_{X} \Rightarrow \hat{\theta}_{X}=\frac{1}{2}
$$

Finally for firm $I Y$ we have

$$
\begin{aligned}
\Pi_{I Y}\left(\rho_{Y}\right) & =\left[A Y \cdot \hat{\theta}_{Y}+B \cdot \hat{\theta}_{h}\right] \rho_{Y} \\
\frac{\partial \Pi_{I Y}}{\partial \rho_{Y}} & =\frac{A Y}{2 \lambda_{Y}}\left\{\lambda_{Y}+y-2 \rho_{Y}\right\}+\frac{B}{\lambda_{B}+\lambda_{Y}-\lambda_{X}}\left\{b-x-\lambda_{X}-2 \rho_{Y}+\lambda_{B}\right\} \\
& =\frac{3 A Y}{4 \lambda_{Y}}\left(\lambda_{Y}-\rho_{Y}\right)+\frac{B}{\lambda_{B}+\lambda_{Y}-\lambda_{X}}\left(\frac{1}{2} \lambda_{Y}-\frac{3}{2} \rho_{Y}+\lambda_{B}-\lambda_{X}\right)
\end{aligned}
$$


This can be solved to give the optimal margin for $I Y$ to charge:

$$
\rho_{Y}=\lambda_{Y}+\underbrace{\left(\lambda_{B}-\lambda_{X}-\lambda_{Y}\right)}_{\text {-ve }} \frac{2}{3\left(1+\frac{A Y}{B} \frac{\lambda_{B}+\lambda_{Y}-\lambda_{X}}{2 \lambda_{Y}}\right)}
$$

We only need the fact that $\lambda_{B}<\lambda_{X}+\lambda_{Y}$ to deduce that $\rho_{Y}<\lambda_{Y}$ therefore

$$
\rho_{Y}<y<\lambda_{Y} \Rightarrow \hat{\theta}_{Y}>\frac{1}{2}
$$

Our maintained assumption that some $B$ consumers purchase hybrids requires $\frac{1}{2}=\hat{\theta}_{X}<\hat{\theta}_{h}$. This is possible if and only if

$$
\begin{aligned}
\hat{\theta}_{X}\left(\lambda_{B}+\lambda_{Y}-\lambda_{X}\right) & <\hat{\theta}_{h}\left(\lambda_{B}+\lambda_{Y}-\lambda_{X}\right) \\
\Leftrightarrow \frac{1}{2}\left(\lambda_{B}+\lambda_{Y}-\lambda_{X}\right) & <b-x-\lambda_{X}-\rho_{Y}+\lambda_{B} \\
\Leftrightarrow \rho_{Y} & <\lambda_{B}-\lambda_{X}
\end{aligned}
$$

Now use $\lambda_{B}=\max \left\{\lambda_{X}, \lambda_{Y}\right\}$. If $\lambda_{X} \geq \lambda_{Y}$ then $\lambda_{B}=\lambda_{X}$ and our requirement would be that $\rho_{Y}<0$ which is impossible. Therefore restrict attention to the case $\lambda_{X}<\lambda_{Y}$; hence $\lambda_{B}=\lambda_{Y}$ and our restriction becomes

$$
\lambda_{X}<\lambda_{Y}-\rho_{Y}=\frac{2 \lambda_{X}}{3\left(1+\frac{A Y}{B} \frac{2 \lambda_{Y}-\lambda_{X}}{2 \lambda_{Y}}\right)}<\frac{2}{3} \lambda_{X}
$$

Another contradiction. Hence hybrid bundles cannot form part of the equilibrium as stated.

Part 2 now follows as we have shown that an interior equilibrium cannot have $B$ consumers forming hybrid bundles, hence they must buy both products from $I I$ or from $I X$ and $I Y$.

For part 3 we establish when the prices given in Lemma 1 do not create an incentive for $B$ consumers to form the hybrid bundle $\langle I I X, I Y\rangle$. This is the relevant deviation as $\hat{\theta}_{X}<\frac{1}{2}<\hat{\theta}_{Y}$. Note that $\langle I I X, I Y\rangle \succ\langle I X, I Y\rangle \Leftrightarrow \theta>\hat{\theta}_{X}$. Therefore our use of $\langle I X, I Y\rangle$ as the alternative to $I I$ is valid if $\hat{\theta}_{B}<\hat{\theta}_{X}$. The required condition is therefore

$$
\begin{aligned}
\frac{\rho_{X}+\rho_{Y}-\lambda_{Y}}{\lambda_{X}+2 \lambda_{Y}} & >\frac{\rho_{X}-\lambda_{X}}{2 \lambda_{X}} \\
\left(2 \lambda_{Y}-\lambda_{X}\right) \rho_{X}-2 \lambda_{X} \rho_{Y} & <\lambda_{X}^{2}
\end{aligned}
$$

Note that if $\lambda_{X}=\lambda_{Y}=\lambda$ for any population splits then $\rho_{X}=\rho_{Y}=\lambda$ and so the left hand side would equal $-\lambda^{2}$ which is strictly less than $\lambda^{2}$, the right hand side.

Proof of Proposition 2. Using the fact that $\lambda_{X}<\lambda_{Y}$, (7) implies that $\rho_{X}>\lambda_{X}$ and so (6) implies $\rho_{X}>x>\lambda_{X} \cdot \lambda_{X}$ is the pre convergence case yielding the price and market share results for $A X$. The margin charged to $A Y$ consumers is given by $\rho_{Y}<\lambda_{Y}$ from (7). (6) then implies that $\rho_{Y}<y<\lambda_{Y}$. For $B$ consumers note that (7) implies that $\rho_{X}+\rho_{Y}>\lambda_{X}+\lambda_{Y}$. Therefore (6) implies that $\rho_{X}+\rho_{Y}>b>\lambda_{X}+\lambda_{Y}$. 
For the profit calculation insert the first order condition for $I Y$ (analogous to 12) into the profit function for $I Y$ (analogous to 5). This yields

$$
\Pi_{I Y}^{\mathrm{partial}}=\rho_{Y}^{2}\left[\frac{A Y}{2 \lambda_{Y}}+\frac{B}{2 \lambda_{Y}+\lambda_{X}}\right]<\frac{\lambda_{Y}}{2}\left[A Y+\frac{B}{1+\frac{\lambda_{X}}{2 \lambda_{Y}}}\right]<\frac{\lambda_{Y}}{2}[A Y+B]=\Pi_{I Y}^{\mathrm{pre}}
$$

Proof of Lemma 3. First let us assume one stop shopping so that all $B$ consumers always go to only one store. The game is now symmetric and so we consider symmetric equilibria. The margins $x$ are only paid by type $A X$ consumers, $b$ by type $B$ and so on. Thus the model collapses to a standard Hotelling one with linear costs for each consumer type. This has a unique equilibrium as the profit functions are negative quadratics in price (a result of the uniform distribution) and so (10) is the Hotelling equilibrium.

Now relax the one stop shop assumption. At any symmetric price equilibrium firms $I$ and II share the market. We wish to show that should firm $I$ lower its component price by some small $\varepsilon>0$ then no type $B$ consumers would decide to create hybrid bundles. Suppose $I$ were to lower the margin on $Y$ by $\varepsilon$. Buying both goods from $I$ gives a higher utility to $\langle I I X, I Y\rangle$ for all type $B$ consumers with

$$
\theta \leq \frac{\lambda_{X}}{\lambda_{B}-\lambda_{Y}+\lambda_{X}}+\frac{x+y-b}{\lambda_{B}-\lambda_{Y}+\lambda_{X}}-\frac{\varepsilon}{\lambda_{B}-\lambda_{Y}+\lambda_{X}}=1+\frac{x+y-b}{\lambda_{X}}-\frac{\varepsilon}{\lambda_{X}}
$$

As $\lambda_{B}=\lambda_{Y}$ (by assumption of the model), $b \leq x+y$ and for small $\varepsilon, I$ is preferred to $\langle I I X, I Y\rangle$ if $\theta \leq 1$. Similarly the bundle from firm $I I$ is preferred to the hybrid bundle $\langle I I X, I Y\rangle$ for small $\varepsilon$ if $\theta \geq \frac{1}{2}$. Thus in any small deviation from symmetric prices the type $B$ consumers would one stop shop. Therefore the price equilibrium found assuming one stop shopping is the only possible price equilibrium of the more general setting.

\section{B Analysis of General Economies of Scope}

Proof of Proposition 5. The pre-convergence case is clear. Consider partial convergence under the assumption that $B$ consumers do not form hybrid bundles. Repeating the proof of Lemma 1 yields unchanged margins for the merged firm at

$$
x=\frac{1}{2}\left(\lambda_{X}+\rho_{X}\right) ; y=\frac{1}{2}\left(\lambda_{Y}+\rho_{Y}\right) ; b=x+y
$$

The independent firms $I X$ and $I Y$ set margins given by

$$
\left(\begin{array}{c}
\rho_{X} \\
\rho_{Y}
\end{array}\right)=\left(\begin{array}{c}
\lambda_{X} \\
\lambda_{Y}
\end{array}\right)+\frac{\beta}{\Delta}\left(\begin{array}{c}
\frac{1}{2} \beta\left(\lambda_{Y}-\lambda_{X}\right)+\left(\frac{3}{2} \alpha_{Y}+\beta\right)\left(\lambda_{B}-\lambda_{X}\right) \\
\frac{1}{2} \beta\left(\lambda_{X}-\lambda_{Y}\right)+\left(\frac{3}{2} \alpha_{X}+\beta\right)\left(\lambda_{B}-\lambda_{Y}\right)
\end{array}\right)
$$


where $\Delta$ is the appropriate determinant:

$$
\Delta=\frac{9}{4}\left(\alpha_{X} \alpha_{Y}+\beta\left(\alpha_{X}+\alpha_{Y}\right)\right)+2 \beta^{2}
$$

and $\alpha_{X}=\frac{A X}{2 \lambda_{X}} ; \alpha_{Y}=\frac{A Y}{2 \lambda_{Y}} ; \beta=\frac{B}{\lambda_{X}+\lambda_{Y}+\lambda_{B}}$ as before.

Now note that $\rho_{X}>\lambda_{X}$ as $\lambda_{Y} \geq \lambda_{X}$ and $\lambda_{B} \geq \lambda_{Y}$ by assumption. Clearly if $\lambda_{X} \approx \lambda_{Y}$ then good $Y$ prices can rise above the pre-convergence benchmark of $\lambda_{Y}$. Whereas if economies of scope are strong so that $\lambda_{B} \approx \lambda_{Y}$ then good $Y$ prices fall below the pre-convergence benchmark. As $y=\frac{1}{2}\left(\lambda_{Y}+\rho_{Y}\right)$ firm $I I$ moves its component $Y$ price in the same direction as $I Y$, though not as far. Next $\rho_{X}+\rho_{Y}>\lambda_{X}+\lambda_{Y}$ so that the bundle price rises. [A local equilibrium as described here can exist for some parameter values using the same derivation used for (9)].

To show that $B$ consumers cannot form hybrid bundles in equilibrium return to the proof of Lemma 2. The substitution of (16) into (17) yields that an equilibrium with hybrid purchases requires

$$
\underbrace{\lambda_{Y}+\lambda_{X}-\lambda_{B}}_{\text {+ve }}<\frac{3}{2} \frac{A Y}{B}\left(\lambda_{B}+\lambda_{Y}-\lambda_{X}\right) \underbrace{\left(\frac{\lambda_{B}-\lambda_{X}-\lambda_{Y}}{\lambda_{Y}}\right)}_{- \text {ve }}
$$

which is a contradiction. Thus hybrid equilibria with even modest economies of scope can be ruled out.

Part 3 is immediate from the price results for part 2.

\section{References}

[1] Armstrong and Vickers (2008), "Competitive Nonlinear Pricing and Bundling", mimeo UCL.

[2] Armstrong and Vickers (2001), "Competitive Price Discrimination", RAND Journal of Economics, 32, 579-605

[3] Carlton and Waldman (2002), "The Strategic Use of Tying to Preserve and Create Market Power in Evolving Industries." RAND Journal of Economics, 33, 194-220.

[4] Crawford and Yurukoglu (2007), "The Welfare Effects of Bundling in Multi-Channel Television Markets", mimeo University of Arizona

[5] Granier and Podesta (2008), "Mixed Bundling and Mergers," mimeo University of Montpellier 1 .

[6] Matutes and Regibeau (1992), "Compatibility and Bundling of Complementary Goods in a Duopoly," Journal of Industrial Economics, 40, 37-54. 
[7] Mussa and Rosen (1978), "Monopoly and Product Quality", The Journal of Economic Theory

[8] Nalebuff (2004), "Bundling as an Entry Barrier," Quarterly Journal of Economics, 159-187

[9] Nalebuff (2000), "Competing Against Bundles." In Incentives, Organization, Public Economics, edited by Peter Hammond and Gareth D. Myles, London: Oxford University Press, pp. 323-336.

[10] Reisinger (2006), "Product Bundling and the Correlation of Valuations in Duopoly," mimeo University of Munich

[11] Thanassoulis (2007), "Competitive Mixed Bundling and Consumer Surplus", Journal of Economics and Management Strategy, 16, 437-467

[12] Whiston (1990), "Tying, Foreclosure, and Exclusion." American Economic Review,80, 83759. 